\title{
Surface Shape from Specularities
}

\author{
Jan Erik Solem and Anders Heyden \\ School of Technology and Society \\ Malmö University, Sweden \\ jes@ts.mah.se
}

\begin{abstract}
In this paper a method for reconstructing a specular surface from an image sequence is presented. It is based on tracking both feature points and specularities through the sequence. The feature points are used to calculate the motion of the camera and provide depth information for some points on the boundary of the surface. The specularities give constraints on the surface normal and these constraints are complemented by a smoothness condition in order to estimate the surface shape. The smoothness measure is based on minimizing the surface curvature and a spline representation of the surface is used in the optimization step.

Using the proposed method it is possible to reconstruct completely textureless smooth surfaces. This is shown in both simulated and real experiments.
\end{abstract}

Keywords: Specularities, Surface Estimation, Structrure from Motion, Splines

\section{Introduction}

The problem of estimating both structure and motion from image sequences taken by hand-held video cameras has been treated by many researchers during the past 5 to 10 years, cf. [1], [2], [3]. Significant achievements have been obtained for both feature extraction algorithms, tracking methods and reconstruction methods, but also related methods to display the result as dense depth maps, cf. [1], mesh generation and texture mapping, cf. [4]. However, little emphasis have been put on reconstructing surfaces, especially in the case of specular surfaces.

In this paper we will deal with the problem of reconstructing a specular surface from an image sequence taken by a hand-held video camera. We will assume that the scene also contains some feature points that makes it possible to use standard methods to calculate the camera motion. It will also be assumed that the direction of the light source is known. This direction can easily be obtained from the calculated motion of the camera if the shadow of the camera can be seen in an image. 


\subsection{Previous Work}

An early paper examining the information available from the motion of specularities under known camera motion is [5]. In the area of reconstructing surfaces from specularities some work has been done on recovering surfaces by illuminating them with circular or extended light sources [6]. Some work has been done on reconstructing perfect mirror surfaces by studying reflections of calibrated scenes containing lines passing through points [7]. Work has also been done on estimating the shape of specular surfaces under laboratory conditions using spline surfaces [8]. These methods all require some form of laboratory setup. They use extended light sources, calibrated scenes, controlled camera motion or other constraints. The method proposed in this paper is valid for general camera motion and general specular scenes.

\subsection{Contribution of Paper}

The main contribution of the paper is to propose an algorithm for reconstructing specular surfaces from an image sequence taken by an uncalibrated hand-held video camera. The proposed method doesn't need controlled camera motion or special preparation of the scene, such as introducing special patterns or controlled lighting. Instead, we estimate the motion using state of the art structure from motion methods, including auto-calibration, and use specularities on the surface arising from a natural light source. The only limitation is that the direction of the light source has to be known or estimated during structure and motion recovery.

\section{Background}

\subsection{Camera Model}

The following notations will be used: $\mathbf{X}$ denotes object points in homogeneous coordinates and $\mathbf{x}$ denotes image points in homogeneous coordinates. The focal point will be denoted $\mathbf{c}$, the camera matrix $P$ and $S$ is a smooth surface in $\mathbb{R}^{3}$. We use the standard pin-hole camera model, cf. [3]. The object points are then related to the image points by a matrix multiplication,

$$
\mathbf{x} \sim P \mathbf{X},
$$

where $\sim$ denotes equality up to scale. Given the camera matrix $P$, the line of sight corresponding to the image point $\mathbf{x}$ is given by

$$
\mathbf{r}(\mathbf{x})=\mathbf{c}+\lambda P^{+} \mathbf{x}
$$

where $\mathbf{c}=\mathcal{N}(P)$ denote the focal point, $\lambda$ the depth parameter and $P^{+}$denote the pseudo-inverse of $P$. Hence if a specularity is observed at point $\mathbf{x}$ in a given camera, (2) denotes the possible locations of the surface reflecting the light. 


\subsection{Structure and Motion Estimation}

To determine the shape of a surface with the method we propose it is necessary to know the motion of the camera. This is obtained using structure from motion techniques. Throughout this paper we assume that there are enough features in the scene to determine motion of the camera and the structure of a limited number of feature points. This is done by extracting and tracking feature points through the image sequence. The fundamental matrix, $F$, and the trifocal tensor, $T$ are estimated and an initial affine reconstruction is obtained from the cheriality constraints, cf. [2]. Finally an initial Euclidean reconstruction is obtained [9]. The Euclidean structure and camera motion is then refined using bundle adjustment, cf. $[3,10]$.

\subsection{Constraints}

The condition for specular reflection is that the surface normal bisects the viewing direction and the incident light direction. For a point on a surface $S$ with normal $\mathbf{N}$ and light source direction $\mathbf{L}$, the relation to the specular reflection direction $\mathbf{R}$ can be seen in Figure 1.

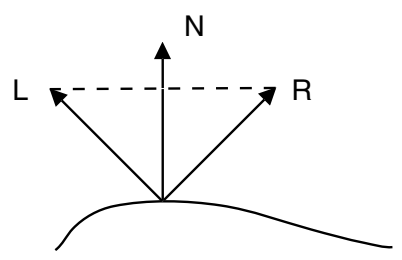

Fig. 1. The condition for specular reflection.

Since we have computed the orientation and position of the camera for the whole sequence and the light source direction can easily be determined (eg. by having one image where the camera shadow is visible) we get a series of constraints on the surface. The constraints are that at the intersection of the ray $\mathbf{r}_{i}$ (2) from the focal point $\mathbf{c}_{i}$ through the specularity in the image $i$ and the surface, the normal $\mathbf{N}_{i}$ is known. This relation is shown in Figure 2. The expression for $\mathbf{N}_{i}$ is then,

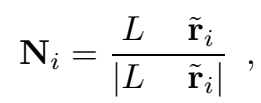

where $\tilde{\mathbf{r}}_{i}$ is the directional vector for each ray, normalized so that $\left|\tilde{\mathbf{r}}_{i}\right|=1$.

The problem is that the depths $\lambda_{i}$ i.e. the distance to the surface from the focal point along $\mathbf{r}_{i}$ can not be determined. Distant light source means that the condition for specularity will be fulfilled at all points on the ray $\mathbf{r}_{i}$.

Further constraints on $\lambda_{i}$ can be added since we have features outside the surface. These were used to compute the camera motion but they also set upper 


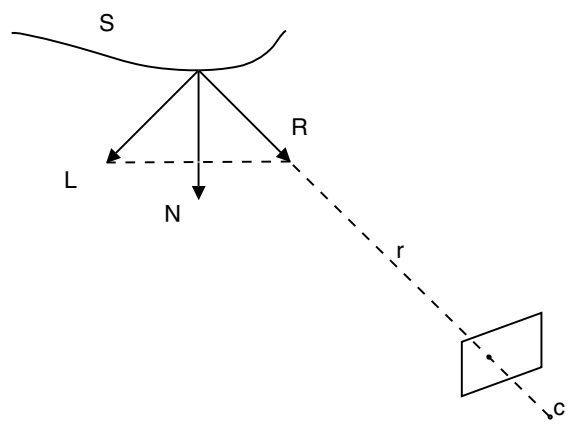

Fig. 2. The relation between a specularity in an image and the surface normal.

and lower bounds on the depths. The surface must lie in a cone defined by the focal point of image $i$ and the non-surface features in image $i$, for all images. The surface must then lie completely in the intersection of all these cones. This volume is called the visual hull, cf. [11].

\section{Proposed Solution}

There are many smooth surfaces at different depths that satisfy (3). We propose to impose extra constraints to find an estimate of the surface. The natural constraint to impose is a smoothness constraint. We have also chosen to represent the surface using a cubic spline representation [12].

We assume that the surface $S$ is a smooth specular surface and that the light source is distant. This means that the light source direction $L$ is a constant vector. By smooth we mean that all components of $S$ have continuous partial derivatives. The proposed algorithm can be summarized as follows:

1. Compute camera motion from background features using structure from motion techniques.

2. Identify specularities in the images and determine the rays $\mathbf{r}_{i}$.

3. Calculate the light source direction $L$ (from camera shadow or other method).

4. Determine the normals $\mathbf{N}_{i}$ using (3).

5. Solve optimization problem, including the smoothness conditions and (3), to obtain the depths $\lambda_{i}$.

6. Interpolate the surface from the result using a spline representation.

\subsection{Optimization Problem}

The gaussian curvature $K$ is independent of surface parametrization and if the surface is parameterized as $S(u, v)=(u, v, f(u, v)), K$ can be expressed in the derivatives $f_{u}, f_{v}, f_{u u}, f_{u v}$ and $f_{v v}$, see [13]: 


$$
K=\frac{f_{u u} f_{v v} \quad f_{u v}^{2}}{1+f_{u}^{2}+f_{v}^{2}} .
$$

Since $f(u, v)$ depend on the depths $\lambda_{i}$, the derivatives and $K$ also depend on $\lambda_{i}$. We are looking for a smooth surface, so we get the following optimization problem:

$$
\min _{\lambda} \int_{S}|K| d u d v
$$

under the constraints

$$
\frac{f_{u}\left(\mathbf{p}_{i}\right) \times f_{v}\left(\mathbf{p}_{i}\right)}{\left|f_{u}\left(\mathbf{p}_{i}\right) \times f_{v}\left(\mathbf{p}_{i}\right)\right|}=\mathbf{N}_{i}, \mathbf{p}_{i} \in \mathbf{r}_{i} \cap S,
$$

There is an inherent ambiguity in the optimization problem, since there are many smooth surfaces that solves the problem above for many different depths, see Figure 3. Since the light source is distant and there is no depth information in the specularities this ambiguity needs to be solved. To solve this we require that we have one or more $3 \mathrm{D}$ points $\mathbf{X}_{j} \in \mathbb{R}^{3}$ on the surface or the surface boundary to position the surface in $\mathbb{R}^{3}$.

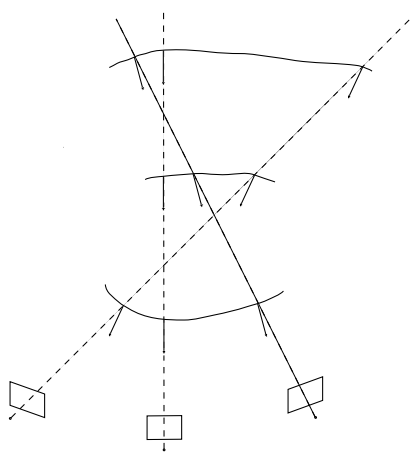

Fig. 3. The surface ambiguity due to unknown depth (note that the ordering of the rays $\mathbf{r}_{i}$ is not constant).

\subsection{Spline Representation}

To reconstruct a surface from the specularities in the image sequence we use a cubic spline representation [12]. The data available are irregularly spaced points $\mathbf{p}_{i}$ on the surface and surface normals $\mathbf{N}_{i}$ at these points. The points are

$$
\mathbf{p}_{i}=\mathbf{c}_{i}+\lambda_{i} \mathbf{r}_{i}
$$


and the normals are given by (3), see Figure 2 .

We assume that the surface can be parameterized as $S(u, v)=(u, v, f(u, v))$. If the surface does not fulfill this condition we can always divide it into surface patches that can be parameterized separately. The condition that $S$ is smooth then just means that $f(u, v)$ has continuous partial derivatives everywhere. The method is as follows:

1. Triangulate the surface points $\mathbf{p}_{i}$ with respect to $u$ and $v$, using Delaunay triangulation.

2. Interpolate the edges in the triangulation using one dimensional cubic interpolation.

3. Subdivide each triangle by adding the midpoint on each edge to the set of data points.

4. Interpolate the normal at the midpoint from the normal values at the endpoints.

5. Repeat 2-4 until desired accuracy is obtained.

\section{Experiments}

The method proposed was tested for both synthetic data and data from real image sequences taken with a digital camcorder. Note that our method only gives the part of the surface inside the convex hull of the $3 \mathrm{D}$-points $\mathbf{X}_{j}$ and surface points $\mathbf{p}_{i}$. The reason for this is that the triangulation in the spline representation gives the convex hull of the points.

\subsection{Synthetic Data}

A synthetic surface $S$ was created by cutting the top of a sphere. The surface was reconstructed using a sequence with 32 cameras and the initial values $\lambda_{0}$ were set to make all surface points $\mathbf{p}_{i}$ lie on a plane. It was also assumed that the coordinates for the corners of the surface were known in order to solve the depth ambiguity from Section 3.1. The result is shown in Figure 4.

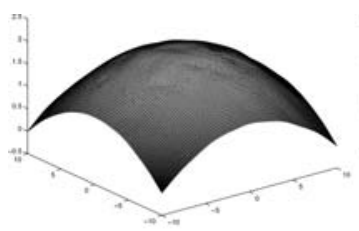

a

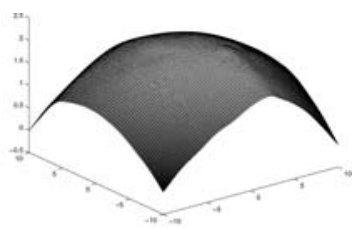

$\mathrm{b}$

Fig. 4. Results from synthetic data, a) true surface, b) surface after 100 iterations. 


\subsection{Real Data}

An image sequence of a car window was taken with a camcorder. Sample images from the sequence are shown in Figure 5. The sequence consisted of 38 images containing specularities and one image of the camera shadow used to determine the light direction. The window has sharp corners which make good features. Two of these were used for determining the surface depth and solving the depth ambiguity. The results were good even if only 38 images were used, see Figure 6 .

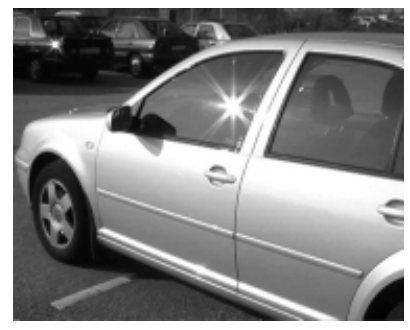

a

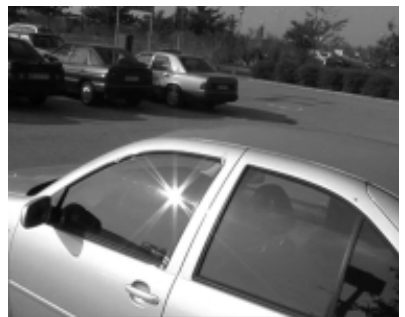

$\mathrm{b}$

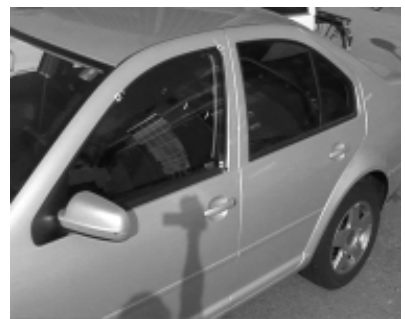

C

Fig. 5. Samples from the image sequence of a car window, a-b) frames number 1 and 20, c) image used to determine the light direction.

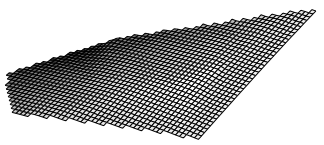

a

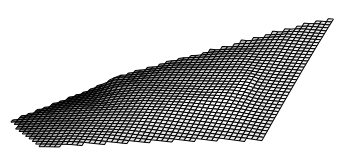

$\mathrm{b}$

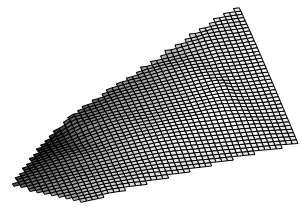

C

Fig. 6. Results for window data. Three views of reconstructed window surface after optimization

\section{$5 \quad$ Summary and Conclusions}

A method for reconstructing a specular surface from an image sequence taken by a hand-held video camera has been presented. The surface is represented using a cubic spline representation that has continuous partial derivatives. The 
method relies on solving an optimization problem incorporating constraints from the specularities in the images and a smoothness constraint on the surface.

Experiments on both synthetic and real data show promising results, even though the images contain noise and the recovery of the scene structure and camera motion is not exact.

\section{Acknowledgments}

The authors would like to thank Martin Johansson for interesting discussions and for help with practical matters.

\section{References}

1. Pollefeys, M., Koch, R., Van Gool, L.: Self-calibration and metric reconstruction in spite of varying and unknown internal camera parameters. In: Int. Conf. Computer Vision, Mumbai, India (1998) 90-95

2. Nister, D.: Automatic Dense Reconstruction from Uncalibrated Video Sequences. PhD thesis, Royal Institute of Technology, KTH (2001)

3. Hartley, R., Zisserman, A.: Multiple View Geometry. Cambridge University Press, The Edinburgh Building, Cambridge CB2 2RU, UK (2000)

4. Morris, D., Kanade, T.: Image-consistent surface triangulation. In: Conf. Computer Vision and Pattern Recognition. Volume 1., Hilton Head SC, USA (2000) 332-338

5. Zisserman, A., Giblin, P., Blake, A.: The information available to a moving observer from specularities. Image and vision computing (1989)

6. Zheng, J.Y., Murata, A.: Acuiring a complete 3d model from specular motion under the illumination of circular-shaped light sources. IEEE Trans. Pattern Analysis and Machine Intelligence 22 (2000) 913-920

7. Savarese, S., Perona, P.: Local analysis for 3d reconstruction of specular surfaces - part ii. In: Proc. European Conf. on Computer Vision. (2002) 759-774

8. Halstead, M.A., Barsky, B.A., Klein, S.A., Mandell, R.B.: Reconstructing curved surfaces from specular reflection patterns using spline fitting of normals. In: Siggraph. (1996) 335-342

9. Heyden, A., Åström, K.: Euclidean reconstruction from image sequences with varying and unknown focal length and principal point. In: Proc. Conf. Computer Vision and Pattern Recognition. (1997) 438-443

10. Triggs, B., McLauchlan, P.F., Hartley, R.I., Fitzgibbon, A.W.: Special sessions bundle adjustment - a modern synthesis. Lecture Notes in Computer Science 1883 (2000) 298-372

11. Laurentini, A.: The visual hull concept for silhouette-based image understanding. IEEE Trans. Pattern Analysis and Machine Intelligence 16 (1994) 913-920

12. Farin, G.: Curves and Surfaces for CAGD: A Practical Guide. Academic Press (2002)

13. Pressley, A.: Elementary Differential Geometry. Springer-Verlag (2001) 